\title{
AN ABSORBING BOUNDARY CONDITION FOR REGULAR AND IRREGULAR WAVE SIMULATIONS
}

\section{BULENT DUZ*, RENE H.M. HUIJSMANS*, ARTHUR E.P. VELDMAN ${ }^{\dagger}$, MART J.A. BORSBOOM ${ }^{\ddagger}$ AND PETER R. WELLENS ${ }^{\ddagger}$}

\author{
*Department of Ship Hydrodynamics \\ Technical University of Delft \\ Mekelweg 2, 2628 CD Delft, The Netherlands \\ e-mail: b.duz@tudelft.nl,r.h.m.huijsmans@tudelft.nl,www.tudelft.nl \\ ${ }^{\dagger}$ Institute for Mathematics and Computer Science \\ University of Groningen \\ P.O. Box 407, 9700 AK Groningen, The Netherlands \\ e-mail: a.e.p.veldman@rug.nl, www.rug.nl \\ $\ddagger$ Deltares \\ P.O. Box 177, 2600 MH Delft, The Netherlands \\ e-mail: mart.borsboom@deltares.nl, peter.wellens@deltares.nl,www.deltares.nl
}

Key words: Absorbing Boundary Condition (ABC), Regular and Irregular Waves

\begin{abstract}
In this paper the use of an absorbing boundary condition (ABC) is investigated for the numerical simulation of regular and irregular waves in three dimensional computational domains where Navier-Stokes equations describe the motion of the fluid. The numerical implementation of the ABC using a staggered grid arrangement is explained in detail. All of the numerical modifications are incorporated into the CFD simulation tool ComFLOW which employs a volume-of-fluid (VOF) method. Numerical examples are provided to demonstrate the performance of the $\mathrm{ABC}$. The reflection character of the $\mathrm{ABC}$ is observed and the results of the computations are discussed and compared.
\end{abstract}

\section{INTRODUCTION}

The CFD simulation of ocean waves remains a challenge even today. Although highly capable numerical features are at the disposal of researchers, particular aspects of numerically solving wave problems in unbounded domains cause various bottlenecks. Typically the phenomena of interest are local but embedded in a vast spatial domain. At this point, the infinite domain, although sometimes it may not be truly unbounded, is truncated via artificial boundaries, thus introducing a finite computational domain and a residual infi- 
nite domain. One of the aforementioned bottlenecks is developing a robust and efficient boundary condition to be imposed on these artificial boundaries.

The Sommerfeld boundary condition [1] was the cornerstone of non-reflecting boundary conditions. Engquist and Majda [2] presented a method to develop the first hierarchy of absorbing boundary conditions. Higdon [3] generalized this theory and showed that Engquist and Majda boundary condition is a subset of the Higdon operators. Since high order boundary operators include high order derivatives both in time and space, Collino and Joly [4] introduced the use of auxiliary variables to circumvent this difficulty. This idea has found widespread interest and has been used by Grote and Keller [5], Givoli and Neta [6], and Hagstrom and Warburton [7] among others. For a general review regarding high order local non-reflecting boundary conditions, see [8].

In this paper we present the derivation of an absorbing boundary condition (ABC) $[9,10]$ along with the numerical implementation of the analytical operator. The $\mathrm{ABC}$ is applied in three dimensional computational domains where a regular Stokes wave and an irregular JONSWAP spectrum wave are traveling under an angle of incidence. Here we focus our attention specifically on the reflection behavior of the $\mathrm{ABC}$ for the duration of the simulations. We end the paper with some concluding remarks.

\section{STATEMENT OF THE PROBLEM}

If we consider water as a homogeneous, incompressible, viscous fluid, we can describe fluid motion in a three-dimensional domain $\Omega$ (see Fig. 1) by the continuity equation and the Navier-Stokes equations in a conservative form as,

$$
\begin{gathered}
\oint_{\Gamma} \mathbf{u} . \mathbf{n} d \Gamma=0, \\
\oint_{\Omega} \frac{\partial \mathbf{u}}{\partial t} d \Omega+\oint_{\Gamma} \mathbf{u} \mathbf{u}^{T} . \mathbf{n} d \Gamma=-\frac{1}{\rho} \oint_{\Gamma}(p \mathbf{n}-\mu \nabla \mathbf{u} . \mathbf{n}) d \Gamma+\oint_{\Omega} \mathbf{F} d \Omega .
\end{gathered}
$$

In (1) and (2), $\Omega$ denotes a volume with boundary $\Gamma$ and normal vector $\mathbf{n}, \mathbf{u}=(u, v, w)^{T}$ is the flow velocity, $\rho$ is the fluid density, $p$ is the pressure, $\mu$ is the dynamic viscosity, $\nabla$ is the gradient operator and $\mathbf{F}=\left(F_{x}, F_{y}, F_{z}\right)^{T}$ represents external body forces acting on the fluid such as gravity.

To solve the above equations in $\Omega$, we impose four types of boundary conditions: a free surface, a wall, an inflow and an absorbing boundary condition. On the west and south boundaries $\Gamma_{W}$ and $\Gamma_{S}$ the incoming wave is prescribed. The incoming wave propagating at an angle $\theta(0<\theta<\pi / 2)$ with the $x$-axis can be simulated by using either a regular wave such as Airy wave or Stokes wave, or an irregular wave such as a superposition of Airy waves. In either case, at every time step starting from $t=0$ free surface elevations, values of the velocity components and the pressure are provided on $\Gamma_{W}$ and $\Gamma_{S}$. At the bottom $\Gamma_{B}$ we specify a no-slip no-penetration condition which is simply the Dirichlet 


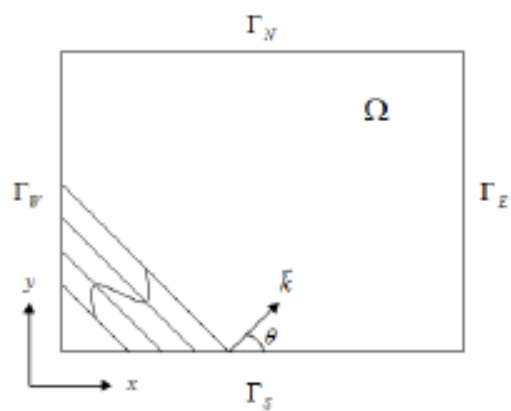

(a)

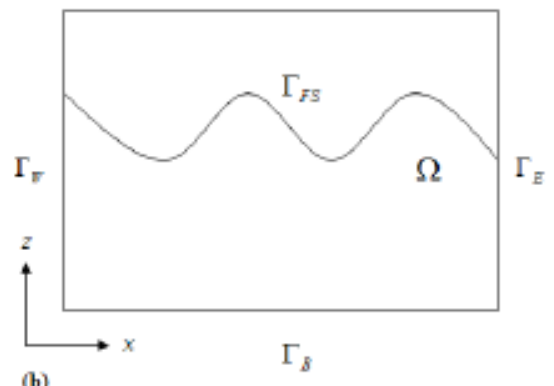

(b)

Figure 1: A computational domain with $\Gamma_{N}$ and $\Gamma_{E}$ as artificial boundaries.

condition, i.e. $\mathbf{u}=0$. At the free surface $\Gamma_{F S}$ resulting from the continuity of normal and tangential stresses the following conditions are implemented for the velocity components and the pressure,

$$
\begin{gathered}
\mu\left(\frac{\partial u_{n}}{\partial t}+\frac{\partial u_{t}}{\partial n}\right)=0, \\
-p+2 \mu \frac{\partial u_{n}}{\partial n}=-p_{0}+\sigma \kappa,
\end{gathered}
$$

where $u_{n}$ and $u_{t}$ correspond to the normal and tangential component of the velocity, respectively, $p_{0}$ is the atmospheric pressure, $\sigma$ is the surface tension and $\kappa$ is the total curvature of the free surface. If we describe the position of the free surface by $s(\mathbf{x}, t)=0$, the displacement of the free surface can be computed via,

$$
\frac{D s}{D t}=\frac{\partial s}{\partial t}+(\mathbf{u} . \nabla) s=0 .
$$

We now introduce two artificial boundaries $\Gamma_{N}$ and $\Gamma_{E}$, see Fig. 1. To complete the statement of the problem we shall employ an $\mathrm{ABC}$ on these artificial boundaries. In this study we will restrict ourselves for a discussion about the behavior of an $\mathrm{ABC}$ in wave simulations where the Navier-Stokes are implemented as the governing equations.

\section{ABSORBING BOUNDARY CONDITION (ABC)}

Consider the following boundary operator on $\Gamma_{E}$ :

$$
\left(\cos \alpha \frac{\partial}{\partial t}+c \frac{\partial}{\partial x}\right) \Phi=0 \text { on } \Gamma_{E}
$$

Higdon [3] showed that (6) is perfectly absorbing if $\alpha$ is equal to the angle of incidence $\theta$ (see Fig. 1(a)) for a wave described by the wave or velocity potential $\Phi$ and traveling with phase speed $c$. Such a wave which satisfies the Laplace equation has the form 


$$
\Phi=\left(C_{1} e^{+k z}+C_{2} e^{-k z}\right) \sin (k x \cos \theta+k y \sin \theta-\omega t+\psi),
$$

where $k$ is the wave number, $\omega$ is the wave frequency and $\psi$ is its phase. The unknowns $C_{1}$ and $C_{2}$ can be determined via the boundary conditions imposed on $\Gamma_{F S}$ and $\Gamma_{B}$.

If we replace $c$ in (6) by the dispersion relation, namely,

$$
c=\sqrt{g h} \sqrt{\frac{\tanh (k h)}{k h}},
$$

we can rewrite (6) as

$$
\left(\cos \alpha \frac{\partial}{\partial t}+\sqrt{g h} \sqrt{\frac{\tanh (k h)}{k h}} \frac{\partial}{\partial x}\right) \Phi=0 .
$$

The boundary condition (9) is perfectly absorbing for this single component but recall that any solution to the Laplace equation for the velocity potential can be represented by a linear superposition of waves which will be referred to as an irregular wave here and elsewhere. Each individual component of this irregular wave has its own frequency, amplitude, wave number and phase. Therefore, the boundary condition (9) cannot annihilate all these wave components simply because it is evidently designed for only one of them.

The corresponding velocity potential of such an irregular wave can be written as

$$
\Phi=\sum_{j=1}^{N}\left(C_{1_{j}} e^{+k_{j} z}+C_{2_{j}} e^{-k_{j} z}\right) \sin \left(k_{j} x \cos \theta+k_{j} y \sin \theta-\omega_{j} t+\psi_{j}\right),
$$

where $N$ denotes the number of modes or components. All flow variables can be calculated by taking derivatives of (10). At this point a question crosses one's mind: Is it possible to develop a boundary condition which has the feature of allowing reflection only to an acceptable threshold for all the wave components which all together form an irregular wave? One can deduce from the way this question is asked that we expect some amount of reflection for such a boundary condition but it will be restricted within certain limits.

Now we introduce the following rational expression which approximates the dispersion relation $(8)$,

$$
c_{a} \approx \sqrt{g h} \frac{a_{0}+a_{1}(k h)^{2}}{1+b_{1}(k h)^{2}},
$$

where a proper choice of coefficients $a_{0}, a_{1}$ and $b_{1}$ would lead to a close approximation for the largest possible range of $k h$ values, see Fig. 2. Thus, reflection from the boundary will be minimized over that specific range of $k h$ values. 


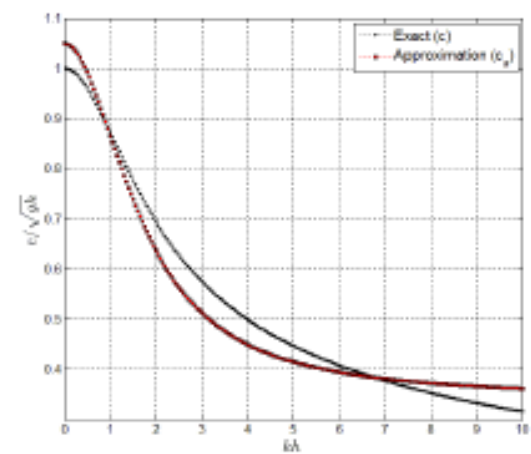

Figure 2: Approximation of the dispersion relation.

As a result of strong effect of dispersion especially in deep water any wave behaves as the sum of a large number of wave components, each traveling at its own dispersive phase speed. To compute these local velocities we will exploit the exponential behavior of (7) and (10) in $z$ direction. After straightforward algebraic manipulations one can derive the following relation

$$
k^{2} \Phi=\frac{\partial^{2}}{\partial z^{2}} \Phi
$$

By employing (12) the dependency of the boundary condition on the wave number is removed since it is calculated using the velocity potential $\Phi$.

Finally we substitute (12) and (11) in (6) to reach the final form of the absorbing boundary condition to be applied on $\Gamma_{E}$

$$
\left(1+b_{1} h^{2} \frac{\partial^{2}}{\partial z^{2}}\right) \cos \alpha \frac{\partial \Phi}{\partial t}+\sqrt{g h}\left(a_{0}+a_{1} h^{2} \frac{\partial^{2}}{\partial z^{2}}\right) \frac{\partial \Phi}{\partial x}=0 \text { on } \Gamma_{E}
$$

Following the same method it is rather easy to write the $\mathrm{ABC}$ on $\Gamma_{N}$.

\subsection{NUMERICAL ALGORITHM}

Since (1) and (2) are specified as the governing equations, the ABC given in (13) must be interpreted in terms of the velocity components and pressure. As we have a staggered grid arrangement for the solution variables inside volume cells (see Fig. 3), the location of the outflow boundary must also be specified appropriately.

We resort to the linearized Bernoulli equation to replace the time derivative of the velocity potential in (13), namely, $\partial \Phi / \partial t=-p_{b}-g z_{p}$. Here and elsewhere the subscript $b$ indicates that the quantity is defined at the outflow boundary and the subscript $p$ indicates that the quantity is evaluated at the elevation of the pressure point within the cell. The spatial derivatives of the velocity potential give the $x-$ and $y$-components of velocity, i.e. $\partial \Phi / \partial x=u_{b}$ and $\partial \Phi / \partial y=v_{b}$, while a further time derivative gives the 


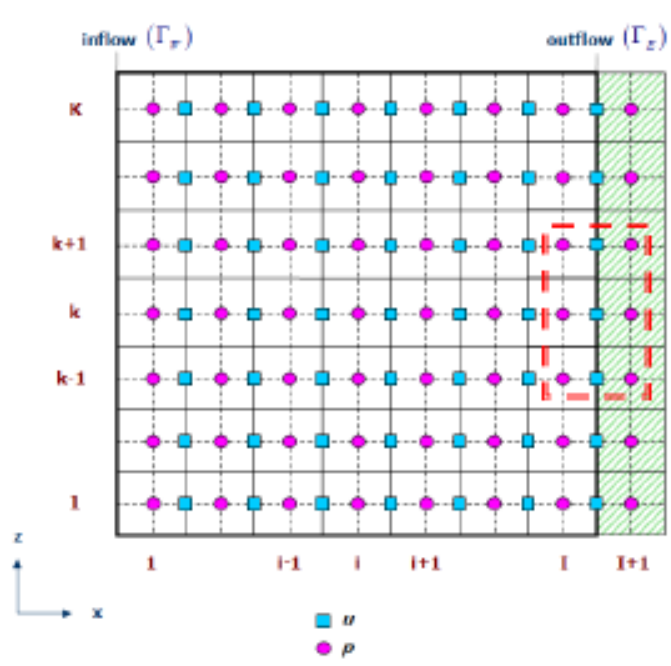

(a) Stencil of the ABC in $x-z$ domain.

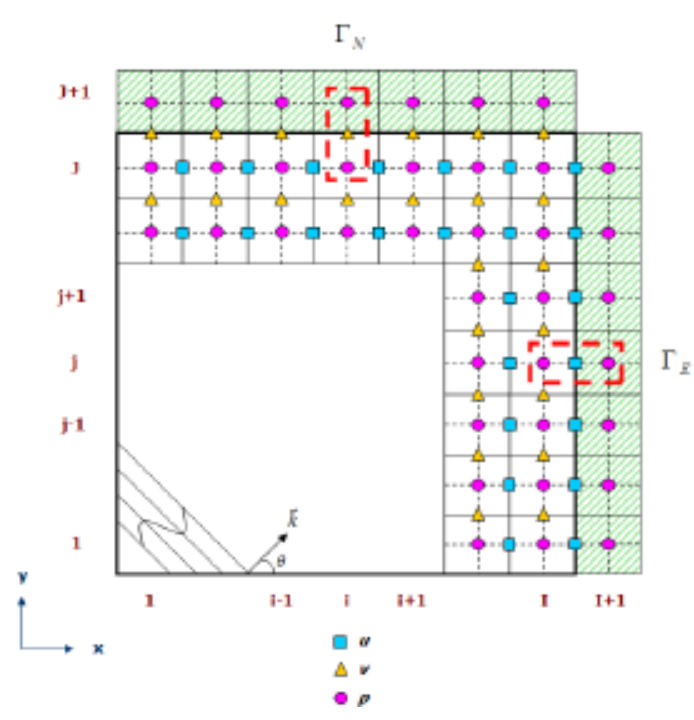

(b) Stencil of the ABC in $x-y$ domain (top view).

Figure 3: Discretization of the $\mathrm{ABC}$ in space

acceleration. Here we will use mirror cells adjacent to the outflow boundary to obtain $p_{b}$ by linear interpolation (see Fig. $3(\mathrm{a}))$, i.e. $p_{b, k}=\left(p_{I+1, k}^{n+1}+p_{I, k}^{n+1}\right) / 2$ for $k=1, \ldots, K$. The shaded area contains the mirror cells which have indices $(I+1, k)$ for $k=1, \ldots, K$. The outflow boundary is situated at the same position along $x$-direction as $u$, therefore we can impose the following, $u_{b}=u_{I, k}^{n+1}$. Note that velocity components and pressure are discretized at the same position on the boundary and also at the same instant in time.

Utilizing the momentum equation (2), the velocity component at the new time step $u^{n+1}$ can be written in terms of the pressure $p^{n+1}$ and the intermediate velocity $\tilde{u}^{n}$ which includes convective and diffusive effects [11]. This modification is necessary to easily plug the $\mathrm{ABC}$ into the pressure Poisson equation which is solved inside the computational domain for the pressure at the new time step $p^{n+1}$. As a result the ABC has the same temporal character as the the pressure Poisson equation. Consequently, we obtain the discrete form of the $\mathrm{ABC}$ to be prescribed on $\Gamma_{E}$ as follows

$$
\begin{aligned}
& {\left[\frac{1}{2} \cos \alpha+a_{0} \sqrt{g h} \frac{\Delta t}{\Delta x_{p(I+1, k)}}+\left(\frac{1}{2} b_{1} h^{2} \cos \alpha+a_{1} h^{2} \sqrt{g h} \frac{\Delta t}{\Delta x_{p(I+1, k)}}\right) \frac{\partial^{2}}{\partial z^{2}}\right] p_{I+1, k}^{n+1} } \\
+ & {\left[\frac{1}{2} \cos \alpha-a_{0} \sqrt{g h} \frac{\Delta t}{\Delta x_{p(I+1, k)}}+\left(\frac{1}{2} b_{1} h^{2} \cos \alpha-a_{1} h^{2} \sqrt{g h} \frac{\Delta t}{\Delta x_{p(I+1, k)}}\right) \frac{\partial^{2}}{\partial z^{2}}\right] p_{I, k}^{n+1} } \\
= & \left(a_{0} \sqrt{g h}+a_{1} h^{2} \sqrt{g h} \frac{\partial^{2}}{\partial z^{2}}\right) \tilde{u}_{I, k}^{n}-g z_{p(I+1, k)} \cos \alpha \quad \text { on } \Gamma_{E},
\end{aligned}
$$

where

$$
\Delta x_{p(I+1, k)}=x_{p(I+1, k)}-x_{p(I, k)} .
$$


Following the same steps, one can easily derive the $\mathrm{ABC}$ on $\Gamma_{N}$. The discrete $\mathrm{ABCs}$ on $\Gamma_{E}$ and $\Gamma_{N}$ are equations for the pressure values in the mirror cells outside the domain, see Fig. 3(b). The stencil for $p_{I+1, k}^{n+1}$ is plotted by a double dashed line in Fig. 3(a). Observing Figs. 3(a) and 3(b) we realize that a typical stencil for a pressure point encompasses 9 flow variables 6 of which reside in the computational domain whereas 3 can be associated with the treatment of the boundary condition.

\section{RESULTS AND DISCUSSIONS}

We compare the results by introducing three error measures,

$$
\begin{gathered}
e(i, j)=\eta_{n}(i, j)-\eta_{r}(i, j), \\
\|e\|_{2}=\sqrt{\sum_{i=1}^{I} \sum_{j=1}^{J}\left(\eta_{n}(i, j)-\eta_{r}(i, j)\right)^{2}}, \\
\|e\|_{\infty}=\max _{i=1,2, \ldots, I} \max _{j=1,2, \ldots, J}\left\{\left|\eta_{n}(i, j)-\eta_{r}(i, j)\right|\right\},
\end{gathered}
$$

where $\eta$ is the free surface elevation. Here the subscript $n$ indicates the numerical results and the subscript $r$ indicates the reference solution. For the regular wave simulation the reference solution is the analytical results arising from the Stokes wave theory. For the irregular wave simulation the reference solution is obtained by solving the problem in a larger domain with the same discretization in space and time.

The pointwise error $e(i, j)$ provides information at particular time instances throughout the simulation. In addition, it demonstrates the exact location of the error in the computational domain which is not the case for the other error measures. The common property of the 2-norm $\|e\|_{2}$ and the infinity norm $\|e\|_{\infty}$ is that they display a complete picture of the error behavior in a single plot. More particularly, we can examine the length of the error vector using $\|e\|_{2}$ whereas $\|e\|_{\infty}$ captures the maximum value in the error vector which is useful especially to check if a certain limit for the error is breached.

\subsection{Results of the regular wave simulation}

A fully developed fifth-order Stokes wave is generated and initialized everywhere in the computational domain at $t=0$ as depicted in Fig. 4. Since we know the exact values of the solution variables for a fifth-order Stokes wave [12], we can compare the numerical results with the theoretical results. The fifth-order Stokes wave with wave height $H=9 \mathrm{~m}$, wave period $T=10 \mathrm{~s}$, wave length $\lambda=161 \mathrm{~m}$, phase speed $c=16.1 \mathrm{~m} / \mathrm{s}$ is simulated by performing 7143 time-steps at $\Delta t=0.007 \mathrm{~s}$. The length and the width of the computational domain is the same, $l_{x}=l_{y}=340 \mathrm{~m}$, and its depth is $l_{z}=179 \mathrm{~m}$ with the water depth of $h=170 \mathrm{~m}$. The grid resolution is $\Delta x \times \Delta y \times \Delta z=2.26 \mathrm{~m} \times 2.26 \mathrm{~m} \times 0.95 \mathrm{~m}$ with $6 \%$ vertical stretching at the free surface. 


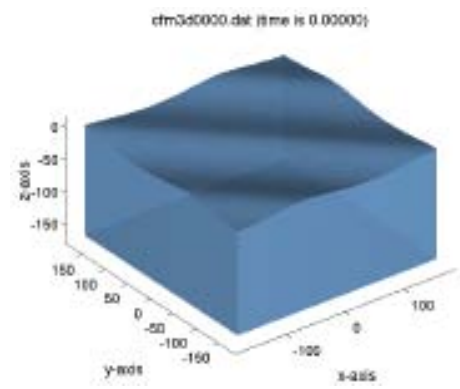

Figure 4: Initial condition for the simulation of the fifth-order Stokes wave. Angle of incidence $\theta=45^{\circ}$.
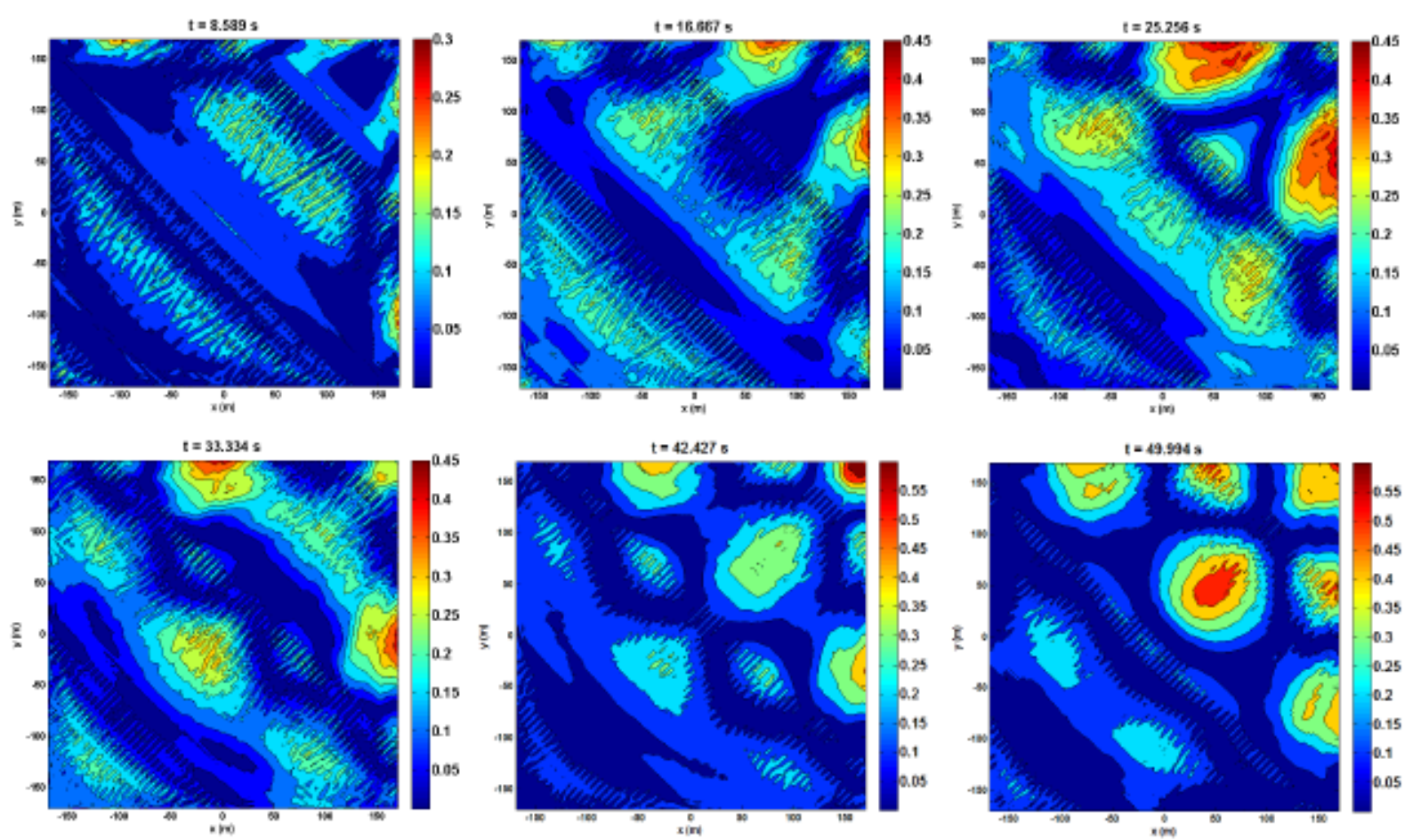

Figure 5: The absolute pointwise errors $e(i, j)$ between the numerical and theoretical results for the simulation of the fifth-order Stokes wave. Errors are shown at times: $t=8.589 \mathrm{~s}, t=16.667 \mathrm{~s}, t=25.256 \mathrm{~s}$, $t=33.334 s, t=42.427 s$ and $t=49.994 s$.

Fig. 5 shows the absolute pointwise errors $e(i, j)$ corresponding to times $t=8.589 \mathrm{~s}$, $t=16.667 \mathrm{~s}, t=25.256 \mathrm{~s}, t=33.334 \mathrm{~s}, t=42.427 \mathrm{~s}$ and $t=49.994 \mathrm{~s}$. The amplitudes of the maximum errors increase in time although not substantially. The reflected waves from the outflow boundaries travel back and perturb the solution in the entire computational domain. Fig. 6 demonstrates the 2-norm $\|e\|_{2}$ and the infinity norm $\|e\|_{\infty}$ both of which are normalized by the wave height. Observing Fig. 5 we notice that large errors are very local whereas in the major part of the domain we have relatively small errors. This is consistent with the fact that $\|e\|_{2}$ has an oscillating character below the maximum value 
of $2.5 \%$ throughout 5 wave periods. Evidently, $\|e\|_{\infty}$ shows a similar behavior but it oscillates generally between the values of $9 \%$ and $4 \%$. For the maximum values of $\|e\|_{\infty}$ we believe we are encountering the effects of reconstruction of the free surface in the VOF algorithm.
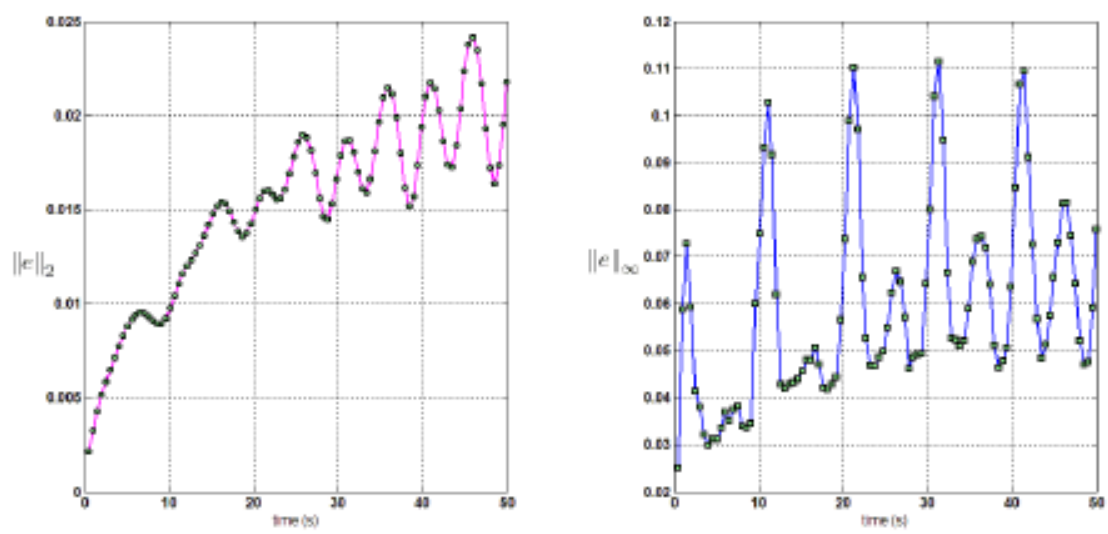

Figure 6: The relative 2-norm $\|e\|_{2}$ and the infinity norm $\|e\|_{\infty}$ in space as a function of time for the regular wave simulation. Both error measures are normalized by the wave height $H=9 \mathrm{~m}$.

\subsection{Results of the irregular wave simulation}

We apply the discrete ABCs to a problem in a three dimensional computational domain where an irregular wave is traveling under an angle of incidence, $\theta=45^{\circ}$. The initial

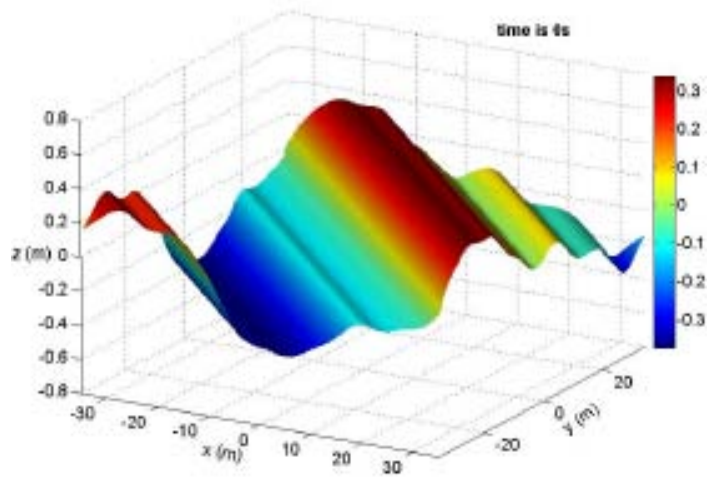

(a) The initial condition for the irregular wave simulation.

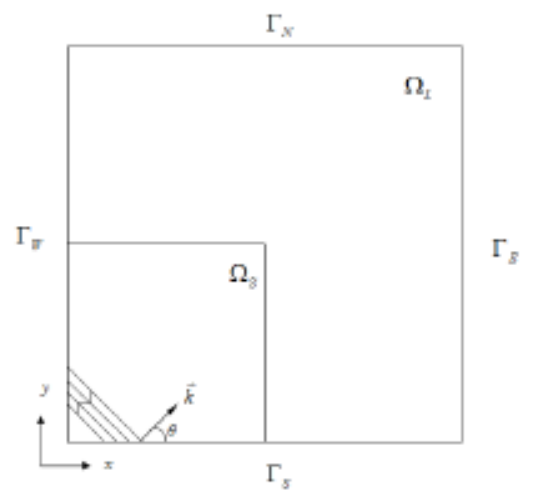

(b) Large domain $\Omega_{L}$ is considered as the reference domain (top view).

Figure 7: The setup for the irregular wave simulations.

condition for the simulation of the irregular wave with 537 Fourier components is shown 
in Fig. 7(a). The domain length in $x$ - and $y$-direction is the same, $l_{x}=l_{y}=70 \mathrm{~m}$ whereas $l_{z}=8 \mathrm{~m}$ with the water depth $h=5 \mathrm{~m}$. The grid resolution is $\Delta x \times \Delta y \times \Delta z=$ $0.28 \mathrm{~m} \times 0.28 \mathrm{~m} \times 0.23 \mathrm{~m}$. A JONSWAP spectrum wave with $T_{p}=10 \mathrm{~s}$ and $H_{s}=1.0 \mathrm{~m}$ is simulated by performing 3964 time-steps at $\Delta t=0.007 \mathrm{~s}$.

As mentioned before, we compute the reference solution by solving the problem in a large domain $\Omega_{L}$ which is twice the size of the small domain $\Omega_{S}$ in in $x$ - and $y$-direction, see Fig. 7 (b) for the illustration of the problem. For each time step, the computational solution in $\Omega_{S}$ is compared to the reference solution in $\Omega_{L}$. In both $\Omega_{S}$ and $\Omega_{L}$ the numerical parameters are the same. Since the flow behavior is highly nonlinear, the linear theory fails to produce correct results under the current circumstances. Therefore, it is not possible to make a comparison with the analytical solution for the irregular wave simulation.
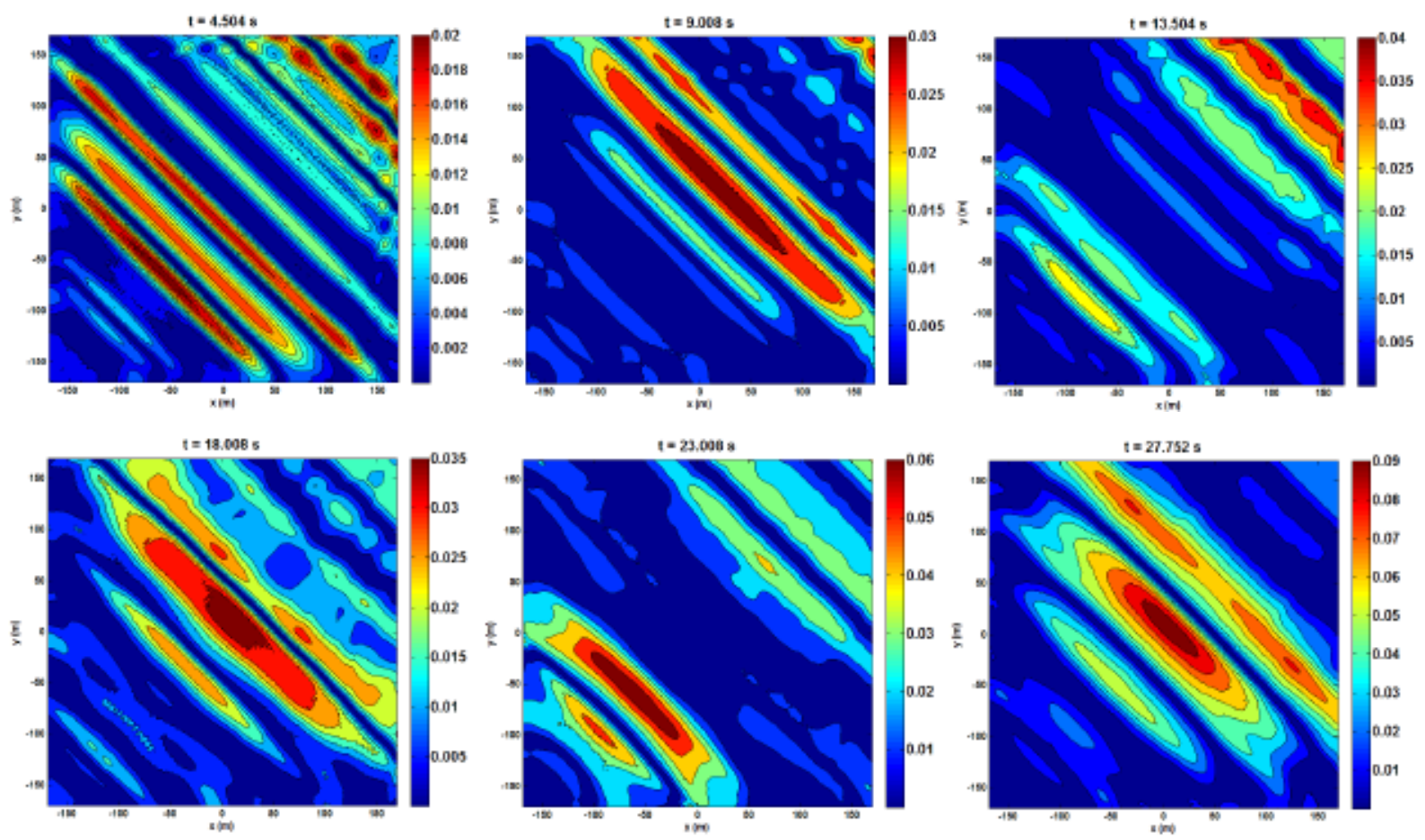

Figure 8: The absolute pointwise errors $e(i, j)$ between the numerical results in large and small domains for the irregular wave simulation. Errors are shown at times: $t=4.504 \mathrm{~s}, t=9.008 \mathrm{~s}, t=13.504 \mathrm{~s}$, $t=18.008 s, t=23.008 s$ and $t=27.752 s$.

In Fig. 8 we demonstrate the absolute pointwise errors $e(i, j)$ corresponding to times $t=4.504 \mathrm{~s}, t=9.008 \mathrm{~s}, t=13.504 \mathrm{~s}, t=18.008 \mathrm{~s}, t=23.008 \mathrm{~s}$ and $t=27.752 \mathrm{~s}$. Compared to the regular wave simulation, large errors cover wider parts in the computational domain, thus $\|e\|_{2}$ has higher values, see Fig. 9. Grid resolution is more significant in irregular wave simulations because short wave components may not be represented well on the grid. 
This is certainly undesirable as these components contribute to the amount of reflection. The characters of $\|e\|_{\infty}$ and $\|e\|_{2}$ are similar as they increase in time (Fig. 9). The error norms show an exponential behavior contrary to the regular wave calculation in which they are oscillatory. This is a result of the absence of a certain beating pattern in irregular waves. Moreover, it should be mentioned that some amount of error is also present in the reference solution although $\Omega_{L}$ is relatively large. Overall, we find deviations of less than $9 \%$ for nearly three wave periods.
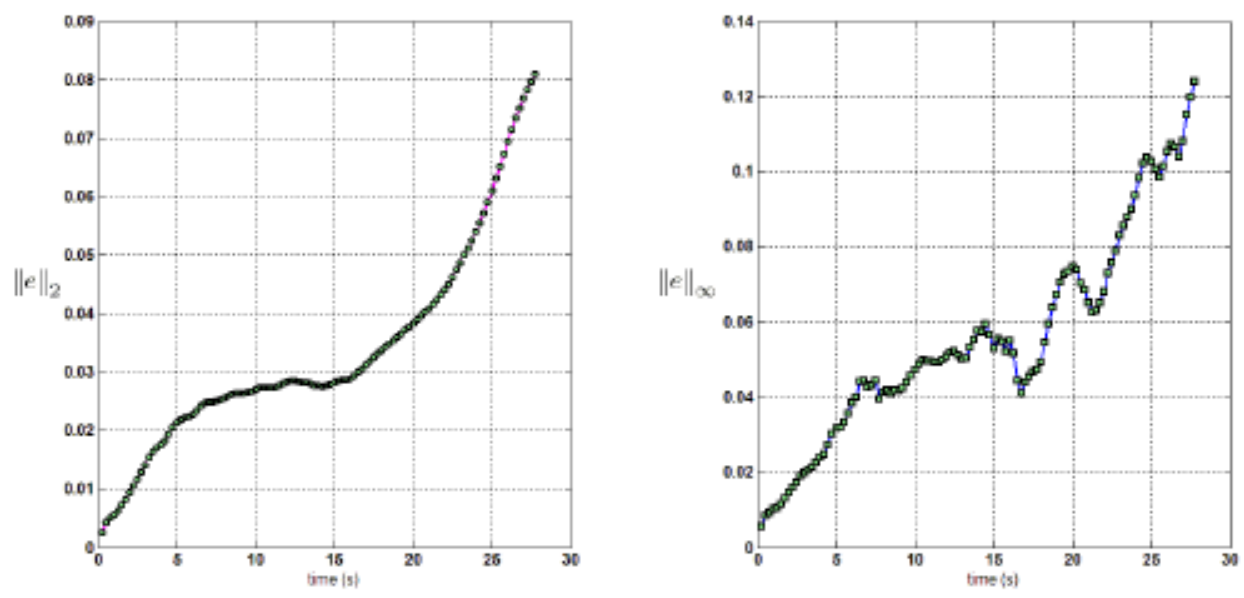

Figure 9: The relative 2-norm $\|e\|_{2}$ and the infinity norm $\|e\|_{\infty}$ in space as a function of time for the irregular wave simulation. Both error measures are normalized by the wave height $H_{s}=1.0 \mathrm{~m}$.

\section{CONCLUDING REMARKS}

In this paper we have presented the derivation and the numerical implementation of an $\mathrm{ABC}$ using the computational framework of the CFD simulation tool ComFLOW. The ABC is applied in three dimensional free surface simulations of regular and irregular waves propagating under an angle of incidence. For this purpose, a fifth-order Stokes wave and a JONSWAP spectrum wave are generated at the inflow boundaries of the computational domains. The results of the numerical computations are compared to various reference solutions to provide sufficient information regarding the performance of the proposed boundary condition. Additionally, the reflection character of the ABC is monitored throughout the calculations and different error measures are exploited to deliver a comprehensive picture for the error behavior.

Overall, the $\mathrm{ABC}$ demonstrated a good performance. In both regular and irregular wave simulations we notice that reflections are less than acceptable thresholds. The numerical results are in reasonable agreement with the reference solutions. Particularly for the irregular wave simulation it would be insightful to observe error behaviors for a longer duration of simulation which will be the subject of the future work. 


\section{Acknowledgment}

This research is supported by the Dutch Technology Foundation STW, applied science division of NWO and the technology programma of the Ministry of Economic Affairs in The Netherlands (contracts GWI.6433 and 10475).

\section{REFERENCES}

[1] Sommerfeld, A. Partial differential equations in physics. Academic Press, 1949.

[2] Engquist, B. and Majda, A. Absorbing boundary conditions for the numerical simulation of waves. Math. Comput. (1977) 31:629-651.

[3] Higdon, R. L. Numerical absorbing boundary conditions for the wave equation. Math. Comput. (1987) 49:65-90.

[4] Collino, F. and Joly, P. New absorbing boundary conditions for the finite element solution of 3D Maxwell's equations. IEEE. Transactions on Magnetics (1995) 31(3):1696-1701.

[5] Grote, M. J. and Keller, J. B. Nonreflecting boundary conditions for time-dependent scattering. Journal Of Computational Physics (1996) 127(1):52-65.

[6] Givoli, D. and Neta, B. High-order non-reflecting boundary conditions for dispersive waves. Wave Motion (2003) 37(3):257-271.

[7] Hagstrom, T. and Warburton, T. A new auxiliary variable formulation of high-order local radiation boundary conditions: corner compatibility conditions and extensions to first-order systems. Wave Motion (2004) 39(4):327-338.

[8] Givoli, D. High-order local non-reflecting boundary conditions: a review. Wave Motion (2004) 39:319-326.

[9] Wellens, P. Wave simulations in truncated domains for offshore applications. PhD Thesis, Technical University of Delft, The Netherlands, 2011.

[10] Duz, B., Huijsmans, R.H.M., Wellens, P. R., Borsboom, M.J.A. and Veldman, A. E. P. Towards a general purpose open boundary condition for wave simulations. In Proc. 30th Conf. on Ocean, Offshore and Arctic Engineering OMAE (2011) OMAE201149979.

[11] Kleefsman, K. M. T., Fekken, G., Veldman, A. E. P., Iwanowski, B., and Buchner, B. A Volume-of-Fluid based simulation method for wave impact problems. J. Comput. Phys. (2005) 206:363-393.

[12] Skjelbreia, L., Hendrickson, J. Fifth order gravity wave theory. In Proc. 7th Conf. of Coastal Engineering (1961) 184-196. 